\title{
PRIMES AND PRIME IDEALS IN SHORT INTERVALS
}

\section{LOÏC GRENIÉ, GIUSEPPE MOLTENI AND ALBERTO PERELLI}

Abstract. We prove the analog of Cramér's short intervals theorem for primes in arithmetic progressions and prime ideals, under the relevant Riemann hypothesis. Both results are uniform in the data of the underlying structure. Our approach is based mainly on the inertia property of the counting functions of primes and prime ideals.

§1. Introduction. A famous theorem of Cramér [2] states that, assuming the Riemann hypothesis, there is always a prime between $x$ and $x+h$ provided $x \geqslant x_{0}$ and $c_{1} \sqrt{x} \log x \leqslant h \leqslant x$, with suitable constants $x_{0}, c_{1}>0$. Actually, under the same assumptions, we have that

$$
\pi(x+h)-\pi(x) \geqslant c_{2} \frac{h}{\log x},
$$

with a suitable $c_{2}>0$, and also that

$$
\pi(x+h)-\pi(x) \sim \frac{h}{\log x}
$$

provided $x \geqslant h=\infty(\sqrt{x} \log x)$. Here $f(x)=\infty(g(x))$ means that $f(x) /$ $g(x) \rightarrow \infty$ as $x \rightarrow \infty$. Apart from the explicit values of the involved constants, this is still the best known result about primes in short intervals, under the Riemann hypothesis. Sharper results can be obtained assuming various forms of the pair-correlation conjecture for the zeta zeros; see, e.g., Heath-Brown [7], Languasco et al [13] and the literature quoted there. A simple proof of Cramér's theorem can be obtained from a suitable smoothed explicit formula for $\psi(x)$; see the footnote of Ingham [9, p. 256].

In this paper we show that rather general theorems of Cramér's type follow, under the appropriate Riemann hypothesis, from two results often already available in the literature, namely a short intervals mean-square estimate and a Brun-Titchmarsh-type theorem. Indeed, the latter result implies that the relevant counting function satisfies a suitable inertia property, which is then played against the short intervals mean-square bound to get a contradiction if the interval is not too short. We illustrate our approach in the case of primes in arithmetic progressions and of prime ideals, since apparently these results do not appear in the literature. In the first case all the ingredients are already known, so we proceed directly to the proof of Cramér's theorem for arithmetic progressions. In the case of algebraic number fields we first deal with the required ingredients; 
see in particular Proposition 1 below, which is of some independent interest. In both cases our results are uniform in the data of the underlying structure. However, in the second case the inertia method gives Proposition 3, which in the uniformity aspect is weaker than Theorem 2, proved here by the classical smoothed explicit formula approach. This is due to the lack, in the current literature, of sharp uniform bounds of Brun-Titchmarsh type for number fields. We shall discuss this issue later on in the paper.

As usual, for $(a, q)=1$ we write

$$
\pi(x ; q, a)=\sum_{\substack{p \leqslant x \\ p \equiv a(\bmod q)}} 1
$$

and let $\varphi(q)$ denote Euler's function. Moreover, given an algebraic number field $K$ of degree $n_{K}$, we denote by $d_{K}$ the absolute value of its discriminant, by $\mathfrak{P}$ the prime ideals of the ring $\mathcal{O}_{K}$ of the integers of $K$, by $N(\mathfrak{P})$ their norm and write

$$
\pi_{K}(x)=\sum_{N(\mathfrak{P}) \leqslant x} 1 .
$$

Finally, given an integer $q \geqslant 1$ and a number field $K$, we denote by GRH and DRH the Riemann hypothesis for the Dirichlet $L$-functions associated with the characters $\chi(\bmod q)$ and for the Dedekind zeta function $\zeta_{K}(s)$, respectively. With this notation, our main results are as follows.

THEOREM 1. Let $(a, q)=1$ and assume GRH. Then there exist absolute constants $x_{0}, c_{1}, c_{2}>0$ such that for $x \geqslant x_{0}$ and $c_{1} \varphi(q) \sqrt{x} \log x \leqslant h \leqslant x$ we have

$$
\pi(x+h ; q, a)-\pi(x ; q, a) \geqslant c_{2} \frac{h}{\varphi(q) \log x} .
$$

Clearly, under the same assumptions the same argument also gives

$$
\pi(x+h ; q, a)-\pi(x ; q, a) \sim \frac{h}{\varphi(q) \log x}
$$

provided $x \geqslant h=\infty(\varphi(q) \sqrt{x} \log x)$.

THEOREM 2. Assume DRH for the number field $K$. Then there exist absolute constants $x_{0}, c_{1}, c_{2}>0$ such that for $x \geqslant x_{0}$ and $c_{1}\left(n_{K} \log x+\log d_{K}\right) \sqrt{x} \leqslant$ $h \leqslant x$ we have

$$
\pi_{K}(x+h)-\pi_{K}(x) \geqslant c_{2} \frac{h}{\log x} .
$$

As before, the same proof shows also that

$$
\pi_{K}(x+h)-\pi_{K}(x) \sim \frac{h}{\log x}
$$

provided $x \geqslant h=\infty\left(\left(n_{K} \log x+\log d_{K}\right) \sqrt{x}\right)$. Note that Theorem 2 represents an instance of Lang's [12] "recipe" asserting that, broadly speaking, when 
extending to a number field $K$ the classical results known for $\mathbb{Q}$ one should replace $\log x$ by $n_{K} \log x+\log d_{K}$. Note also that if $K$ is a cyclotomic field then the quality of the $K$-uniformity in Theorem 2 is comparable to the $q$-uniformity in Theorem 1.

We conclude by remarking that the technique in the proof of the above theorems works for rather general counting functions, giving individual short intervals results as soon as suitably sharp short intervals mean-value and inertiatype results are available.

§2. Proofs. As customary, we prove Theorem 1 for the $\psi$-function and then the required result is recovered by elementary arguments, since $h / \varphi(q)$ is large enough. Let $X$ be sufficiently large, $q, h \leqslant X,(a, q)=1$ and write

$$
\Delta(x, h)=\psi(x+h ; q, a)-\psi(x ; q, a)-\frac{h}{\varphi(q)} .
$$

The required mean-square bound follows from a result of Prachar [15] under GRH (see also Goldston and Yıldırım [5]), namely

$$
\int_{X}^{2 X}|\Delta(x, h)|^{2} d x \ll h X \log ^{2}(q X),
$$

where the constant in the $\ll$-symbol is absolute. Let now $h / \varphi(q)>X^{1 / 10}$. From the well-known Brun-Titchmarsh theorem, see Montgomery and Vaughan [14], we deduce that if there exists $\bar{x} \in(X, 2 X)$ such that

$$
|\Delta(\bar{x}, h)|>\frac{1}{4} \frac{h}{\varphi(q)},
$$

then

$$
|\Delta(x, h)|>c \frac{h}{\varphi(q)}
$$

for all $x \in\left(\bar{x}-c^{\prime} h, \bar{x}+c^{\prime} h\right)$, with certain absolute constants $c, c^{\prime}>0$. Inequalities (3) and (4) express the inertia property of the $\psi$-function (see also Bazzanella and Perelli [1, Theorem 1]).

Let now

$$
E(X, h)=\left\{x \in[X, 2 X]:|\Delta(x, h)|>\frac{1}{4} \frac{h}{\varphi(q)}\right\}
$$

and suppose that $E(X, h) \neq \emptyset$. Then from (2)-(4) we get

$$
h\left(\frac{h}{\varphi(q)}\right)^{2} \ll \int_{X}^{2 X}|\Delta(x, h)|^{2} d x \ll h X \log ^{2}(q X) ;
$$

thus, $h \ll \sqrt{X} \varphi(q) \log (q X)$. Hence, with suitable absolute constants in the $\gg$-symbols and provided $x$ is sufficiently large,

$$
\psi(x+h ; q, a)-\psi(x ; q, a) \gg \frac{h}{\varphi(q)}
$$

if $x \geqslant h \gg \varphi(q) \sqrt{x} \log (q x)$. Theorem 1, and the statement after it, therefore follow. 
As anticipated in the Introduction, in the number fields case we first present the proof of a weaker form of Theorem 2 in the uniformity aspect, obtained by the inertia approach. Write

$$
\psi_{K}(x)=\sum_{N\left(\mathfrak{P}^{m}\right) \leqslant x} \log N(\mathfrak{P})=\sum_{n \leqslant x} \Lambda_{K}(n),
$$

say, and

$$
\Delta_{K}(x, h)=\psi_{K}(x+h)-\psi_{K}(x)-h
$$

and let $L=\log X$. The analog of (2) is given by the following result.

Proposition 1. Assume DRH for the number field $K$. Then there exist absolute constants $c, X_{0}>0$ such that for $X \geqslant X_{0}$ and $2 \leqslant h \leqslant X$ we have

$$
\int_{X}^{2 X}\left|\Delta_{K}(x, h)\right|^{2} d x \leqslant c X\left(h+L^{2}\right)\left(n_{K} L+\log d_{K}\right)^{2} .
$$

Proof. Let $2 \leqslant T \leqslant x$; the constants in the $O$ - and $\ll$-symbols below are absolute. Denoting by $N_{K}(T)$ the number of zeros $\rho=\beta+i \gamma$ of $\zeta_{K}(s)$ with $0 \leqslant \beta \leqslant 1$ and $|\gamma| \leqslant T$, using the notation in the Introduction, we unconditionally have

$$
N_{K}(T)=\frac{n_{K}}{\pi} T \log T+\frac{T}{\pi} \log \left(\frac{d_{K}}{(2 \pi e)^{n_{K}}}\right)+O\left(\log \left(d_{K} T^{n_{K}}\right)\right) ;
$$

see Kadiri and $\mathrm{Ng}$ [10]. Moreover, using (5) in the explicit formula in Lagarias and Odlyzko [11] (specialized to the case of $\zeta_{K}(s)$ ) we have, again unconditionally, that

$$
\psi_{K}(x)=x-\sum_{|\gamma| \leqslant T} \frac{x^{\rho}}{\rho}+R_{K}(x, T),
$$

with

$$
R_{K}(x, T)=O\left(\frac{x}{T}\left(n_{K} \log x+\log d_{K}\right) \log x\right) .
$$

Proposition 1 follows now from (4) and (6) by the classical arguments in Saffari and Vaughan [16, Lemmas 5 and 6] (notice a misprint in (6.20) there, where $h^{2}$ on the right-hand side should be replaced by $h$ ); here is a brief sketch. Arguing as in [16, Lemma 6], we have (see (6.21) there)

$$
\begin{aligned}
& \int_{X}^{2 X}\left|\Delta_{K}(x, h)\right|^{2} d x \\
& \quad \ll \frac{X}{h} \int_{h / 3 X}^{3 h / X}\left(\int_{X}^{3 X}\left|\psi_{K}(x+\theta x)-\psi_{K}(x)-\theta x\right|^{2} d x\right) d \theta .
\end{aligned}
$$

Choosing $T=X$ in (6), the contribution of $R_{K}(x, T)$ to the right-hand side of (7) is

$$
\ll X L^{2}\left(n_{K} L+\log d_{K}\right)^{2},
$$


while the contribution of the remaining part of the explicit formula is, thanks to $(5)$,

$$
\begin{aligned}
& \ll X^{2}\left(\frac{h}{X}\right)^{2} N_{K}\left(\frac{X}{h}\right) \max _{2 \leqslant t \leqslant X / h}\left(N_{K}(t+1)-N_{K}(t)\right) \\
& \ll h X\left(n_{K} L+\log d_{K}\right)^{2} .
\end{aligned}
$$

Proposition 1 follows from (7)-(9).

Proposition 1 represents another instance of Lang's "recipe" reported in the Introduction. As far as we know, such a phenomenon has not been established in the case of Brun-Titchmarsh-type bounds, and actually it is not clear to us how the right extension should look like in this case; we briefly discuss this issue at the end of the section. Hence, we use the following simple but uniform bound, which however is unlikely to be sharp in the range needed here.

Proposition 2. Let $K$ be a number field and $2 \leqslant h \leqslant x$. Then

$$
\pi_{K}(x+h)-\pi_{K}(x) \leqslant 4 n_{K} \frac{h}{\log h} .
$$

Proof. Again we use the notation in the Introduction. Let $\left\{k_{j}\right\}_{j \in J}, k_{j} \geqslant 1$, be the exponents of the prime powers in the interval $(x, x+h]$; clearly, $|J| \leqslant h+1$. Since it is well known that for $1 \leqslant k \leqslant n_{K}$ there are at most $n_{K} / k$ prime ideals of $\mathcal{O}_{K}$ with norm $p^{k}$, we have

$$
\pi_{K}(x+h)-\pi_{K}(x) \leqslant n_{K} \sum_{j \in J} \frac{1}{k_{j}}\left(\pi\left((x+h)^{1 / k_{j}}\right)-\pi\left(x^{1 / k_{j}}\right)\right) .
$$

But $(x+h)^{1 / k}-x^{1 / k} \leqslant x^{1 / k} h /(k x)$; hence, applying to $\pi\left((x+h)^{1 / k_{j}}\right)-\pi\left(x^{1 / k_{j}}\right)$ the Brun-Titchmarsh theorem when $k_{j}=1$ (Montgomery and Vaughan [14] with modulus $q=1$ ) and the trivial bound $\leqslant h /\left(k_{j} \sqrt{x}\right)+1$ when $k_{j} \geqslant 2$, we get

$$
\pi_{K}(x+h)-\pi_{K}(x) \leqslant 2 n_{K} \frac{h}{\log h}+n_{K} \sum_{j \in J, k_{j} \geqslant 2} \frac{1}{k_{j}}\left(\frac{h}{\sqrt{x}} \frac{1}{k_{j}}+1\right) .
$$

Since clearly $\sum_{j \in J, k_{j} \geqslant 2} 1 / k_{j} \leqslant \log (|J|+1) \leqslant \log (h+2)$, Proposition 2 follows by a simple computation.

Proposition 3. Theorem 2 holds with $c_{1} n_{K}^{1 / 2}\left(n_{K} \log x+\log d_{K}\right) \sqrt{x} \leqslant$ $h \leqslant x$ in place of $c_{1}\left(n_{K} \log x+\log d_{K}\right) \sqrt{x} \leqslant h \leqslant x$.

Proof. We argue along the lines of Theorem 1. Indeed, for $X$ sufficiently large and, e.g., $h / n_{K}>X^{1 / 10}$, from Proposition 2 we have that if there exists $\bar{x} \in(X, 2 X)$ with

$$
\left|\Delta_{K}(\bar{x}, h)\right|>\frac{1}{4} h \text { then }\left|\Delta_{K}(x, h)\right|>c h
$$


for all $x \in\left(\bar{x}-c^{\prime} h / n_{K}, \bar{x}+c^{\prime} h / n_{K}\right)$, with certain absolute constants $c, c^{\prime}>0$. Playing this against Proposition 1, we therefore obtain that

$$
\frac{h^{3}}{n_{K}} \ll\left(n_{K} L+\log d_{K}\right)^{2} h X .
$$

Hence, $h \ll n_{K}^{1 / 2}\left(n_{K} L+\log d_{K}\right) \sqrt{X}$, and Proposition 3 follows.

The sharper result stated in Theorem 2 is obtained using the direct approach by the smoothed explicit formula. We follow the general lines of the proofs in Dudek [3] and Dudek et al [4], where explicit versions of Ingham's approach to Cramér's theorem are developed. Integrating the infinite explicit formula for $\psi_{K}(x)$ from 2 to $x$, see Grenié and Molteni [6, (1.3a) and Lemmas 3.2 and 3.3], we obtain

$$
\int_{2}^{x} \psi_{K}(t) d t=\frac{x^{2}}{2}-\sum_{\rho} \frac{x^{\rho+1}}{\rho(\rho+1)}-c_{K} x+c_{K}^{\prime}+O\left(n_{K} x \log x\right),
$$

where $\rho$ runs over the non-trivial zeros of $\zeta_{K}(s)$ and $c_{K}, c_{K}^{\prime}$ are certain constants depending on $K$; we are not concerned with their values, since $c_{K}$ and $c_{K}^{\prime}$ simply disappear after the manipulations leading to the next displayed equation. Introducing the weight $w(n)=\max (1-|x-n| / h, 0)$ as in the last row of $[3$, p. 773] and arguing as on p. 774 there, we get

$$
\begin{aligned}
W(x, h) & :=\sum_{x-h<n<x+h} \Lambda_{K}(n) w(n) \\
& =h-\frac{1}{h} \sum_{\rho} \frac{(x+h)^{\rho+1}-2 x^{\rho+1}+(x-h)^{\rho+1}}{\rho(\rho+1)}+O\left(n_{K} \frac{x}{h} \log x\right) .
\end{aligned}
$$

Now we split the sum over the $\zeta_{K}$-zeros into the subsums $\Sigma_{1}$ and $\Sigma_{2}$ cutting at $T=x / h$, and use DRH and (5) as in the proof of [3, Theorem 1.2], thus obtaining

$$
W(x, h)=h+O\left(\left(n_{K} \log x+\log d_{K}\right) \sqrt{x}\right)+O\left(n_{K} \frac{x}{h} \log x\right) .
$$

From (11), we obtain the behavior of the unweighted sum, observing that for every $0<\varepsilon<1$,

$$
\begin{aligned}
& -\frac{1}{\varepsilon}((1-\varepsilon) W(x,(1-\varepsilon) h)-W(x, h)) \\
& \quad \leqslant \psi_{K}(x+h)-\psi_{K}(x-h) \\
& \quad \leqslant \frac{1}{\varepsilon}((1+\varepsilon) W(x,(1+\varepsilon) h)-W(x, h)),
\end{aligned}
$$

since $\Lambda_{K}(n) \geqslant 0$. Theorem 2 and the assertion after it follow at once. 
We conclude with a brief discussion on the Brun-Titchmarsh theorem for number fields and its relevance to this paper. Note that the dependence on the data of $K$ in Proposition 2, where bounded $h$ are allowed, is essentially best possible. Indeed, if a prime $p \in(x, x+2]$, say, splits in $\mathcal{O}_{K}$ into the product of $n_{K}$ prime ideals of norm $p$, then clearly $\pi_{K}(x+2)-\pi_{K}(x) \geqslant n_{K}$, while Proposition 2 gives $\pi_{K}(x+2)-\pi_{K}(x) \leqslant c n_{K}$, with some absolute $c>0$. Note that, although the constant in the classical Brun-Titchmarsh theorem is of great interest, the absolute constant in front of $n_{K}$ in (10) plays essentially no role in this paper. For larger $h$ the dependence on $K$ in (10) is unsatisfactory, as indeed the prime ideal theorem, or (1), shows.

The bounds of Brun-Titchmarsh type are usually obtained by the Selberg sieve. Apparently, an application of the Selberg sieve to $\pi_{K}(x+h)-\pi_{K}(x)$, see, e.g., Hinz and Loedemann [8], brings into play the residue $v_{K}$ of the Dedekind zeta function $\zeta_{K}(s)$. It is well known that $v_{K}$ depends on several invariants of $K$, and even under DRH its dependence on such invariants is not completely under control. This adds some difficulties to the problem of obtaining sharp versions of Proposition 2. Perhaps one can prove that

$$
\pi_{K}(x+h)-\pi_{K}(x) \leqslant c h / \log \left(h / d_{K}\right),
$$

but this is weaker than what is obtainable for an abelian extension $K / \mathbb{Q}$, namely

$$
\pi_{K}(x+h)-\pi_{K}(x) \leqslant c h / \log \left(h / q_{K}\right),
$$

where $q_{K}$ is the conductor of $K$. Bound (12) can be obtained by coupling the classical Brun-Titchmarsh theorem for arithmetic progressions with the Kronecker-Weber theorem for abelian extensions of $\mathbb{Q}$. Actually, when (12) is coupled with Proposition 1, we get back, in the abelian case, a result of the same quality as Theorem 1.

Acknowledgements. We wish to thank Olivier Ramaré for detecting some inaccuracies in a previous version. This research was partially supported by grant PRIN2015 Number Theory and Arithmetic Geometry. The authors are members of the INdAM groups GNSAGA and GNAMPA.

\section{References}

1. D. Bazzanella and A. Perelli, The exceptional set for the number of primes in short intervals. J. Number Theory 80 (2000), 109-124.

2. H. Cramér, Some theorems concerning prime numbers. Ark. Mat. Astr. Fys. 15 (1920), 1-33.

3. A. W. Dudek, On the Riemann hypothesis and the difference between primes. Int. J. Number Theory 11 (2015), 771-778.

4. A. W. Dudek, L. Grenié and G. Molteni, Primes in explicit short intervals on RH. Int. J. Number Theory 12 (2016), 1391-1407.

5. D. A. Goldston and Y. Yıldırım, Primes in short segments of arithmetic progressions. Canad. J. Math. 50 (1998), 563-580.

6. L. Grenié and G. Molteni, Explicit smoothed prime ideals theorems under GRH. Math. Comput. 85 (2016), 1875-1899.

7. D. R. Heath-Brown, Gaps between primes, and the pair correlation of zeros of the zeta-function. Acta Arith. 41 (1982), 85-99. 
8. J. Hinz and M. Loedemann, On Siegel zeros of Hecke-Landau zeta-functions. Monatsh. Math. 118 (1994), 231-248.

9. A. E. Ingham, On the difference between consecutive primes. Q. J. Math. 8 (1937), 255-266.

10. H. Kadiri and N. Ng, Explicit zero density theorems for Dedekind zeta functions. J. Number Theory 132 (2012), 748-775.

11. J. C. Lagarias and A. M. Odlyzko, Effective versions of the Chebotarev density theorem. In Algebraic Number Fields (ed. A. Fröhlich), Academic Press (London, 1977), 409-464.

12. S. Lang, On the zeta function of number fields. Invent. Math. 12 (1971), 337-345.

13. A. Languasco, A. Perelli and A. Zaccagnini, An extension of the pair-correlation conjecture and applications. Math. Res. Lett. 23 (2016), 201-220.

14. H. L. Montgomery and R. C. Vaughan, The large sieve. Mathematika 20 (1973), 119-134.

15. K. Prachar, Generalisation of a theorem of A. Selberg on primes in short intervals. In Topics in Number Theory (Colloquia Mathematica Societatis János Bolyai 13) (ed. P. Turán), North-Holland (Amsterdam, 1976), 267-280.

16. B. Saffari and R. C. Vaughan, On the fractional parts of $x / n$ and related sequences. II. Ann. Inst. Fourier 27 (1977), 1-30.

Loïc Grenié,

Dipartimento di Ingegneria Gestionale, dell'Informazione e della Produzione,

Università di Bergamo,

viale Marconi 5,

24044 Dalmine (BG),

Italy

E-mail: loic.grenie@gmail.com

Alberto Perelli,

Dipartimento di Matematica,

Università di Genova,

via Dodecaneso 35,

16146 Genova,

Italy

E-mail: perelli@dima.unige.it
Giuseppe Molteni,

Dipartimento di Matematica, Università di Milano, via Saldini 50, 20133 Milano,

Italy

E-mail: giuseppe.molteni1@unimi.it 